\title{
The Glutaminase Inhibitor Compound 968 Exhibits Potent in vitro and in vivo anti-tumor Effects in Endometrial Cancer
}

\section{Lingqin Yuan ( $\nabla$ ylq_2020@163.com )}

Shandong Tumor Hospital and Institute https://orcid.org/0000-0002-2087-8852

Hui Guo

Shandong Tumor Hospital and Institute

Guoyou Pan

Shandong Tumor Hospital and Institute

Cong Wang

Shandong Tumor Hospital and Institute

Dapeng Li

Shandong Tumor Hospital and Institute

Naifu Liu

Shandong Tumor Hospital and Institute

Ling Wei

Shandong Tumor Hospital and Institute

Peipei Li

Shandong Tumor Hospital and Institute

Xiugui Sheng

Shandong Tumor Hospital and Institute

Primary research

Keywords: Endometrial cancer, Compound 968, GLS1, mTOR, growth, paclitaxel

Posted Date: July 28th, 2020

DOI: https://doi.org/10.21203/rs.3.rs-47882/v1

License: (9) This work is licensed under a Creative Commons Attribution 4.0 International License.

Read Full License 


\section{Abstract}

Background: Glutamine is one of the primary nutrients utilized by cancer cells for energy production and biosynthesis. Interfering with glutamine metabolism may impose anti-tumor effects. In this study, we aimed to investigated the anti-tumorigenic effects of GLS1 inhibition in endometrial cancer cells in vitro and in vivo.

Methods: In this study, we assessed the relationship between glutaminase-1 enzyme (GLS1) expression and prognosis in endometrial cancer by bioinformatics analyses. Overall survival (OS) and progressionfree survival (PFS) analyses were performed using the Kaplan-Meier method. The effects of compound 968 on cell proliferation, cell cycle, apoptosis, cellular stress, invasion, and AKT/mTOR/S6 pathway inhibition in human endometrial cancer cell lines were assessed. The in vivo therapeutic potential of compound 968 in endometrial cancer was evaluated using tumor xenografts.

Results: We found that GLS1 expression is elevated during endometrial cancer progression and is associated with poor prognosis. The GLS1-targeting compound 968 was able to reduce cancer cell proliferation, induce cell cycle arrest at the $\mathrm{G} 1$ phase, inhibit cell invasion, as well as promote cellular stress and cancer cell apoptosis. Compound 968 treatment significantly increased the sensitivity of cells to paclitaxel. Moreover, the treatment of endometrial cancer cells with compound 968 resulted in AKT/mTOR/S6 signaling pathway inhibition. In xenograft mouse models of endometrial cancer, compound 968 significantly suppressed tumor growth.

Conclusion: We conclude that compound 968 is a promising anti-tumorigenic agent and that combination with paclitaxel may be a valuable strategy for the treatment of endometrial cancer.

\section{Background}

Endometrial cancer is the most common gynecologic malignancy in the developed world, with both its incidence and mortality have increased over the past few decades[1]. Although surgery is used effectively to manage early-stage endometrial cancer, advanced-stage patients and patients with recurrent disease are usually treated with comprehensive approaches, and their prognosis remains poor. It is, therefore, imperative to explore novel adjuvant treatment approaches for endometrial cancer.

Glutamine, the most abundant amino acid in plasma, is one of the main nutrients utilized by cancer cells for biosynthesis. Glutamine conversion into tricarboxylic acid (TCA) cycle metabolites, also known as glutaminolysis, has been established as a hallmark of cancer metabolism[2, 3]. Glutamine represents a primary carbon source supporting the TCA cycle and energy production and denotes nitrogen for the synthesis of many non-essential amino acids, nucleotides, and hexosamines needed to support the fastproliferating cancer cells[4]. Glutamine is also involved in glutathione synthesis, maintaining the redox balance. In addition, glutamine, together with leucine, actives mTORC1 signaling in tumor cells via a Rag GTPase- dependent mechanism[5]. We have previously found that glutamine activates the mTOR/S6 
pathway in ovarian cancer[6]; hence, targeting glutamine metabolism may provide a promising therapeutic strategy for cancer.

GLS is the rate-limiting amidohydrolase that catalyzes the first step glutamine conversion to glutamate during glutaminolysis. Mammalian cells contain two different genes encoding for GLS: the kidney-type (GLS1) and liver-type (GLS2) enzymes. Moreover, two major splice variants of GLS1 exist in humans, a long form (referred to as KGA) and a short form (GAC). Both isoforms are expressed in kidneys, as well as in several other tissues, including tumors[4, 7]. GLS1 is often upregulated in cancer, supporting tumor cell growth by regulating c-myc, RB1, Rho GTPase, and STAT1, as well as the Raf-MEK-ERK and NF-kB pathways[7-10]. siRNA-mediated GLS1 gene silencing has been shown to impair cancer cell growth in a variety of tumor types, including lymphoma, prostate cancer, breast cancer, glioma, and non-small cell lung cancer[11, 12]. In hepatocellular carcinoma, increased levels of GLS1 have been associated with a more advanced disease stage and poor prognosis[13].

The small molecule 968 is an allosteric inhibitor of GLS1 and inhibits the activity of both KGA and GAC isoforms[4]. The antitumor effects of compound 968 have been demonstrated in a variety of tumor entities that are highly resistant to conventional chemotherapy, including ovarian, brain, pancreatic, and breast cancer[ $[14,15]$. However, there is currently no evidence regarding the effect of compound 968 on cell growth in endometrial cancer. In the present study, we aimed at elucidating the antitumor effects of compound 968 on endometrial cancer cells and in mouse xenograft endometrial cancer models.

\section{Materials And Methods}

\section{GLS1 expression in endometrial cancer}

Publicly available data were retrieved and analyzed in the context of this study. Transcriptomics (microarray) data from 877 cancer cell lines retrieved from the Cancer Cell Line Encyclopedia (CCLE) [http://www.broadinstitute.org/ccle]. RNA sequencing data from uterine corpus endometrial carcinoma (UCEC) patients were downloaded from The Cancer Genome Atlas (TCGA) database using Broad GDAC Firehose [https://gdac.broadinstitute.org]. GLS1 expression levels in different stages of endometrial cancer progression and normal endometrium were assessed using Gene Expression Profiling Interactive Analysis (GEPIA) [http://gepia.cancer-pku.cn/index.html].

\section{Cell culture and reagents}

The human endometrial cancer cell lines Ishikawa and HEC-1B were purchased from the China Center for Type Culture Collection (Wuhan, China). The Ishikawa cell line was maintained in MEM medium (Gibco, Invitrogen, Carlsbad, CA, USA) supplemented with $5 \%$ fetal bovine serum (FBS) (Gibco, Invitrogen). HEC1B cells were maintained in RPMI 1640 medium (Gibco, Invitrogen, Carlsbad, CA, USA) supplemented with $5 \%$ FBS. All cell culture media were supplemented with $100 \mathrm{U} / \mathrm{ml}$ of penicillin and $100 \mu \mathrm{g} / \mathrm{ml}$ of streptomycin. Cells were cultured in a humidified $5 \% \mathrm{CO}_{2}$ atmosphere at $37^{\circ} \mathrm{C}$. 
Compound 968 was purchased from Calbiochem (Billerica, MA) and diluted with DMSO to make up stock solutions of $10 \mathrm{mmol} / \mathrm{L}$. The anti-GLS1 antibody was purchased from Abcam (1:1000), and all the other antibodies were obtained from Cell Signaling Technology (1:1000).

\section{Colony formation assay}

Ishikawa and HEC-1B human endometrial cancer cells were harvested at a log phase of growth. Cells were seeded into 6-well plates (800 cells/well) and were incubated for $24 \mathrm{~h}$ in their standard growth media to allow for cell adhesion. After incubation, the cells were treated with compound $968(0,5,10$, and $25 \mu \mathrm{M})$ for $24 \mathrm{~h}$, followed by the replacement of the media with drug-free cell culture media. Cells were further incubated at $37^{\circ} \mathrm{C}$ for 14 days, during which the cell culture media was refreshed every three or four days. After incubation, cells were subjected to $0.5 \%$ crystal violet staining, and colony quantification was achieved by counting under a light microscope. The treatments were performed in triplicates, and the clonogenic assays were repeated three times.

\section{Cell proliferation assay}

The cell viability of human endometrial cancer cells was assessed in MTT assays, as previously described[14]. Briefly, Ishikawa and HEC-1B cells harvested at the logarithmic growth phase were seeded into 96-well plates (4000 cells/well) in regular media and allowed to adhere for $5 \mathrm{~h}$. After the incubation, cells were treated with increasing concentrations of compound 968, ranging between 2 and $50 \mu \mathrm{M}$ for 48 h. Alternatively, cells were incubated in the presence of $2 \mu \mathrm{M}$ or $10 \mu \mathrm{M}$ of compound 968 for $24,48,72$, 96 , and $120 \mathrm{~h}$. The cells were treated with different concentrations of paclitaxel $(0,0.1,1.0,10,50 \mathrm{nM})$ in media with $10 \mu \mathrm{M}$ compound 968 for $72 \mathrm{~h}$. To quantify cell viability, MTT was added in each of the wells of the $96-w e l l$ plate at a final concentration of $0.25 \mathrm{mg} / \mathrm{ml}$. After one hour of incubation, the MTTcontaining cell culture media was replaced by $100 \mu \mathrm{l}$ of DMSO, and the optical absorbance at $570 \mathrm{~nm}$ was measured on a plate reader. The treatments were performed in triplicates, and the experimental procedure was repeated three times.

\section{Annexin-V assay}

The ability of compound 968 to induce cell apoptosis was examined by flow cytometry using the Annexin-V FITC kit (BD Biosciences Pharmingen, San Diego, USA) and following the instructions of the manufacturer. In brief, human endometrial cancer cells were seeded into 6-well plates at $2 \times 10^{5}$ cells/well and allowed to adhere for $5 \mathrm{~h}$. Cells were then exposed to increasing concentrations of compound 968 ( 0 , $5,10,25 \mu \mathrm{M})$. After a 24-hour incubation, the cells were harvested with EDTA-free $0.25 \%$ trypsin and washed with PBS. Subsequently, cells were incubated in $100 \mu \mathrm{l}$ of Annexin-V/PI solution for $15 \mathrm{~min}$ in the dark and analyzed on a FACS Calibur instrument. The flow cytometry results were analyzed using the FlowJo Software 7.6 (TreeStar). At least two experimental repeats were conducted.

\section{Cell cycle analysis}


As described above, $2 \times 10^{5}$ cells/well were seeded into 6 -well plates for $5 \mathrm{~h}$, and then the cells were treated with increasing concentrations of compound 968 for $48 \mathrm{~h}$. After incubation, the cells were harvested using $0.05 \%$ trypsin, washed with phosphate-buffered saline (PBS) solution, and fixed using $90 \%$ methanol; fixed cells were kept at $-20^{\circ} \mathrm{C}$ until they were subjected to cell cycle analysis by flow cytometry. Before flow cytometry, residual methanol was removed by washing with PBS. After the cells were centrifuged, they were incubated in $50 \mu \mathrm{l}$ of RNase A solution $(250 \mu \mathrm{g} / \mathrm{ml})$ containing $10 \mathrm{mM}$ EDTA for $30 \mathrm{~min}$ at $37^{\circ} \mathrm{C}$. Subsequently, cells were resuspended in $50 \mu \mathrm{l}$ of propidium iodide (PI) staining solution, which contained $2 \mathrm{mg} / \mathrm{ml} \mathrm{PI}, 0.1 \mathrm{mg} / \mathrm{ml}$ Azide, and $0.05 \%$ Triton X-100, and incubated for 10 min protected from light. The stained cell samples were analyzed on a FACS Calibur instrument (Becton Dickinson). The flow cytometry results were analyzed using the FlowJo Software, version 7.6. At least two independent repetitions were performed for each condition.

\section{Reactive oxygen species (ROS) generation analysis}

The ability of the compound 968 to affect ROS generation and induce cellular oxidative stress in human endometrial cancer cells was assessed by measuring the intracellular ROS levels using the ROS-sensitive fluorescence indicator DCFH-DA (Jiangsu KeyGEN BioTECH Corp. Ltd, Jiangsu, China) and flow cytometry, as described previously (Eruslanov E and Kusmartev S, 2010). In brief, Ishikawa and HEC-1B cells were seeded in 6 -well plates at $2.5 \times 10^{5}$ cells/well. After cells were allowed to adhere for $5 \mathrm{~h}$, the cells were treated with 5,10 , and $25 \mu \mathrm{M}$ of compound 968 for $12 \mathrm{~h}$. Subsequently, cells were incubated in the presence of DCFH-DA $(20 \mu \mathrm{M})$ for $20 \mathrm{~min}$, and then harvested, washed with PBS, and then analyzed on the FACS Calibur instrument. The flow cytometry results were analyzed using the FlowJo Software, version 7.6. At least two independent repetitions were performed for each condition.

\section{Invasion assay}

The effects of compound 968 treatment on the invasion capacity of human endometrial cancer cells were assessed using transwell chambers with pores of $8 \mu \mathrm{m}$ in dimeter (Costar, NewYork, NY, USA), as standard methodologies described elsewhere[16]. Briefly, serum-starved (for $12 \mathrm{~h}$ ) Ishikawa and HEC-1B cells were seeded into the upper chambers of 24-transwell inserts at 50,000 cells/well, followed by a 12hour incubation in $50 \mu \mathrm{l}$ of serum-free medium; during the incubation, the lower chambers were covered with $150 \mu \mathrm{l}$ of regular cell growth media containing different concentrations of compound 968 . To allow for sufficient time for the invasion into the lower chamber to occur, the plates were incubated for $24 \mathrm{~h}$ at $37^{\circ} \mathrm{C}$. After incubation, a cotton swab was used to remove any cells that did not migrate through the membrane. The cells that had successfully invaded into the lower chamber were fixed and stained using $0.1 \%$ crystal violet. Images from five representative fields were acquired using a light microscope, followed by cell quantification. At least two independent repetitions were performed for each condition.

\section{Western blot analysis}

Total protein was extracted from endometrial cancer cells using RIPA buffer (Cwbiotech, CW2333S, China), and the protein was quantified with the BCA assay kit (Cwbiotech, CW0014S, China). Equal 
amounts $(20 \mu \mathrm{g})$ of the protein were separated in a $12 \%$ SDS-PAGE and transferred onto polyvinylidene fluoride (PVDF) membranes (Millipore, Billerica, MA, USA). The membranes were blocked in $5 \%$ nonfat milk and then incubated overnight at $4^{\circ} \mathrm{C}$ with 1:1000 dilution of primary antibodies. The membranes were washed and incubated with a secondary peroxidase-conjugated antibody for $1 \mathrm{~h}$ at room temperature. The membranes were visualized using ECL blotting detection reagents (Bio-Rad, USA). Relative protein contents were quantified using $\beta$-actin as a loading control. Each experiment was repeated at least twice.

\section{Tumor xenograft growth assay}

Four-week-old BALB/c nude female mice (Beijing HFK Bioscience Co., Ltd., Beijing, China) with severe combined immunodeficiency were used for our experiments. A total of $1 \times 10^{7}$ Ishikawa cells in $0.1 \mathrm{ml}$ of serum-free MEM were injected subcutaneously into the right flank of the mice. Two weeks after injection, the mice were treated with intraperitoneal injections of compound $968(5 \mathrm{mg} / \mathrm{kg})$ every day for two weeks. Subcutaneous tumors were dissected and weighed. Tumor volume was calculated using the following equation: tumor volume $=\left(\right.$ width $^{2} \times$ length $) / 2$. All mice were handled according to protocols approved by the Institutional Animal Care and Use Committee of Shandong Cancer Hospital and Institute, China. All methods were performed in accordance with the $\mathrm{NIH}$ guidelines and regulations.

\section{Immunohistochemical analysis and scoring}

Mouse tumor tissues were formalin-fixed and paraffin-embedded. Slides $(4 \mu \mathrm{m})$ were treated with xylene and rehydrated, and then $3 \%$ hydrogen peroxide was used to eliminate endogenous peroxidase activity. Antigen retrieval was performed through boiling in a microwave, and $5 \%$ bovine serum albumin was applied to block nonspecific binding. Slides were incubated with the primary antibodies of rabbit antiGLS1 (1:1000, Abcam), rabbit anti-phosphor-S6 Ribosomal protein (Ser235/236) (1:1000, Cell Signaling Technology), rabbit anti-cyclinD1 protein (1:1000, Cell Signaling Technology) and rabbit anti-E-cadherin (1:1000, Cell Signaling Technology) at $4^{\circ} \mathrm{C}$ overnight. At least 3 pieces $200 \times$ views were randomly selected for taking pictures each slide in each group. Try to make the tissue fill the whole field of view when taking pictures, make sure that the background light of each photo is same. Use Image-Pro Plus 6.0 software (Media Cybernetics, Inc., Rockville, MD, USA) to select the same brown-yellow color as the uniform standard for judging the positive of all photos, and analyze each photo to get the integrated optical density value (IOD) and positive pixel area (AREA) of each photo. And calculate the average optical density (average optical density, AOD value), AOD = IOD / AREA, the larger the AOD value, the higher the positive expression level.

\section{Statistical analysis}

Uterine Corpus Endometrial Carcinoma (TCGA, PanCancer Atlas; $n=527$ ) RNA sequencing and clinical data were retrieved using Broad GDAC Firehose [https://gdac.broadinstitute.org]. Overall survival and progression-free survival analyses were performed using the Kaplan-Meier method. Statistical significance in differences in survival was determined by the log-rank (Mantel-Cox) test using GraphPad 
Prism version 7.0c. Data are expressed as mean \pm standard error. Comparisons between two groups were performed using the two-tailed Student's $t$-test. Significance was set at ${ }^{*} P<0.05, * * P<0.01$.

\section{Results}

\section{GLS1 expression is elevated during endometrial cancer progression and associates with poor prognosis}

We first investigated the expression of GLS1 mRNA in 877 cancer cell lines from microarray data of CCLE. As shown in (Fig. 1a), GLS1 is highly expressed in endometrial cell lines. Even though its mRNA levels were not significantly higher in endometrial cancer tissues compared to normal tissues (Fig. 1b), within endometrial cancer patients, its expression was elevated during cancer progression from early stages to more advanced stages (Fig. 1c).

Kaplan-Meier survival analysis was conducted to assess the effects of GLS1 expression on survival in endometrial cancer patients. We found that endometrial cancer patients with high GLS1 expression had a significantly worse OS and PFS compared with those with low GLS1 expression (Fig. 1d and1e).

\section{Compound 968 inhibits cell proliferation and colony formation in endometrial cancer cells}

Given that GLS1 expression is associated with endometrial cancer progression and poor prognosis, we tested the effects of GLS1 inhibition on cell growth in endometrial cancer cell lines using MTT assays. Our results indicated that compound 968 inhibited cell proliferation in a dose- and time-dependent manner (Fig. 2a and 2b). The IC50 values was $25 \mu \mathrm{M}$ for the Ishikawa and Hec-1B cells. Furthermore, we demonstrated that $10 \mu \mathrm{M}$ of compound 968 treatment increased the sensitivity of the endometrial cancer cells to paclitaxel (Fig. 2c). We also assessed the long-term effects of GLS1 inhibition by compound 968 on the cell survival of Ishikawa and HEC1-B cells, using in vitro colony formation. Our results indicated that compound 968 resulted in decreased colony formation in a dose-dependent manner (Fig. 2e). Taken together, these results suggested the suppressive effects of compound 968 on cell proliferation and synergistic inhibitory effects in Ishikawa and HEC-1B cells.

Given that compound 968 is an allosteric inhibitor of GLS1, we tested its effects on the expression of GLS1. Ishikawa and HEC-1B cells were treated with compound 968 for $24 \mathrm{~h}$, and the protein levels of GLS1 were assessed by immunoblotting, using an antibody that recognizes both KGA and GAC isoenzymes. We found lower protein levels of GLS1 when cells were treated with compound 968 (Fig. 2f).

\section{Compound 968 induces apoptosis in endometrial cancer cells}

To determine whether the reduction in cell numbers observed after compound 968 treatment was due to apoptosis induction, we treated the two cell lines with increasing concentrations of compound 968 for 24 h. The results showed that, although there were no apparent changes in early apoptosis, high concentrations of compound 968 resulted in an increase in the percentage of cells undergoing late apoptosis in both cell lines (Fig. 3a). This is in agreement with previous studies, which reported that compound 968 induced late apoptosis in less than $20 \%$ of cells at 48 h and 72 h of treatment in the triple- 
negative breast cancer (TNBC) cell line MDA-MB-231[17]. However, a previous study of ovarian cancer indicated that compound 968 increased early apoptosis[14]. We also found that a $24 \mathrm{~h}$ treatment with compound 968 resulted in decreased MCL-1 protein levels in a dose-dependent manner (Fig. 3b). Collectively, these results suggest that compound 968 treatment in endometrial cancer cells induces apoptosis.

\section{Compound 968 affects cell cycle progression in endometrial cancer cells}

Studies have indicated that glutamine is necessary for cell cycle progression for cell cycle from S-phage into cell division[18]. We, therefore, reasoned to examine the effects of compound 968 on cell cycle progression in these two cell lines and found that treatment with compound 968 for $48 \mathrm{~h}$ resulted in significant G1 phase cell cycle arrest and a decrease in the percentage of cells in S-phase (Fig. 4a). Western blotting showed that compound 968 treatment led to reduced cyclin D1 protein levels and increased protein levels of p21 and p27 (Fig. 4b). This was consistent in both cell lines. Our results are consistent with the effects of compound 968 on ovarian cancer cells[14].

\section{Compound 968 induces cell stress and autophagy in endometrial cancer cells}

In addition to its role in cell proliferation, apoptosis, and cell cycle progression, glutamine also participates in maintaining cellular redox homeostasis. To test whether sensitivity to GLS1 inhibition correlates with increased oxidative stress upon compound 968 treatment, we measured the ROS levels in Ishikawa and HEC-1B cells using DCFH-DA. Treatment of cells with compound 968 for $24 \mathrm{~h}$ resulted in increased ROS levels in both cell lines in a dose-dependent manner. In both cell lines, the intracellular ROS production was increased by more than 1.4 -fold at a dose of $25 \mu \mathrm{M}$ compared with the control cells (Fig. 5a). We also treated the cells with $10 \mu \mathrm{M}$ of compound 968 for different times and found that the protein levels of protein kinase-like endoplasmic reticulum kinase (PERK) and calnexin and binding immunoglobulin protein (BIP) increased in a time-dependent manner (Fig. 5b), suggesting that treatment with compound 968 induced cellular stress in endometrial cancer cells.

Autophagy is a homeostatic mechanism for maintaining cell survival by recycling organelles and macromolecules[19]. Autophagy is known to have a dual role in tumorigenesis, which depends on the type and stage of cancer, as well as the tumor microenvironment. The induction of autophagy is characterized by the upregulation of the microtubule-associated protein light chain 3 (LC3) and Beclin-1. The effects of compound 968 on autophagy in both cell lines were examined. Ishikawa and HEC-1B cells were treated with different concentrations of compound 968 for $24 \mathrm{~h}$. Western blotting showed that compound 968 markedly induced LC3-II and Beclin-1 elevation at the protein level in a dose-dependent manner (Fig. 5c). This finding was consistent in both cell lines.

\section{Compound 968 inhibits cell invasion in endometrial cancer cells}

To address whether GLS1 inhibition plays a role in regulating the migration of cancer cells, we investigated the influence of compound 968 on cell migration by a transwell assay. We found that 
compound 968 significantly inhibited endometrial cancer cell invasion in a dose-dependent manner after $24 \mathrm{~h}$ of treatment (Fig. 6a). Cell invasion is mediated by a variety of membrane proteins as well as the modulation of cytoskeletal assembly. To further dissect the mechanism by which the compound 968 affects cell motility and migration of endometrial cancer cells, the expression levels of E-cadherin, $\beta$ catenin, and vimentin were analyzed by western blotting. After $24 \mathrm{~h}$ of treatment, cells were found to have increased protein levels of E-cadherin, while $\beta$-catenin and vimentin protein levels were lower (Fig. 6b). Taken together, these data suggest that compound 968 can inhibit the invasion of endometrial cancer cells.

\section{Compound 968 inhibits the AKT/mTOR/S6pathway in endometrial cancer cells}

Given that the hyper-activation of PI3K/AKT/mTOR pathway strongly associates with tumor progression and metastasis in endometrial cancer[20], we evaluated the effects of compound 968 on the mTOR pathway. Ishikawa and HEC-1B cells were treated with various concentrations of compound 968 for $24 \mathrm{~h}$. We found that P-S6 levels were significantly decreased by compound 968 treatment in a dose-dependent manner in both cell lines (Fig. 7a), suggesting that the downstream targets of mTOR were inhibited. We also found that compound 968 was able to reduce P-AKT473 levels after $24 \mathrm{~h}$ of treatment (Fig. 7a). These data suggest that compound 968 may show its anti-tumorigenic activity via inhibiting the $\mathrm{AKT} / \mathrm{mTOR} / \mathrm{S} 6$ signaling pathway in endometrial cancer cells.

\section{Compound 968 inhibits tumor growth in a xenograft mouse model}

To examine the relevance of GLS1 inhibition in tumor growth in vivo, we evaluated the effects of compound 968 on tumor growth in Ishikawa tumor-bearing mice. The mice were allocated into two groups ( $n=15$ per group) and were treated with intraperitoneal injections of compound 968 ( 5 $\mathrm{mg} / \mathrm{kg} / \mathrm{day}$ ) or placebo for two weeks. The mice showed no overt signs of toxicity and maintained normal activity during the treatment period. After two weeks of treatment, the tumors were removed, photographed, and weighed. Tumor burden was decreased by $36.7 \%$ after treatment with compound 968 compared to the control group (Fig. 8b). Additionally, treatment with compound 968 resulted in a significant suppression in tumor growth, as the tumor volume was decreased by a 1.8-fold compared to the control group (Fig. 8a). These data suggest that compound 968 effectively suppresses the endometrial tumor growth in vivo.

To further investigate the anti-tumorigenic activity and mechanisms of compound 968 in vivo, the expression levels of GLS1, p-S6, cyclin D1, and E-cadherin in tumor tissues were evaluated by immunohistochemistry (Fig. 8c). The expression of GLS1 was slightly reduced following treatment compared to that observed in control tumors. Moreover, compound 968 treatment resulted in decreased cyclin D1 expression. Consistent with our in vitro results, compound 968 treatment resulted in obviously decreased p-S6 levels, suggesting that compound 968 inhibits tumor growth in vivo through the mTORC1 pathway. Additionally, E-cadherin levels were higher in mice treated with compound 968 , further supporting the role of compound 968 in suppressing invasion of endometrial cancer cells. 


\section{Discussion}

Accumulating evidence suggests that GLS1 is upregulated in several cancers. Bioinformatics analyses using RNA sequencing data obtained through the TCGA revealed that GLS1 expression is elevated during endometrial cancer progression. We also found that its over-expression is associated with poor prognosis in endometrial cancer patients. Considering that glutamine is an important source of energy for cancer cells and one of the main nutrients for biosynthesis, targeting GLS1 has recently emerged as a promising approach for cancer treatment. Although compound 968 has been demonstrated to exert anti-tumor effects in different cancer entities, no previous reports have addressed the therapeutic potential of compound 968 in endometrial cancer. In this study, we investigated the anti-tumor effects of compound 968 in endometrial cancer both in vitro and in vivo. Our results demonstrated that targeting glutamine metabolism using compound 968 inhibited cell proliferation, induced cell cycle arrest at G1 phase, and induced apoptotic cell death in the Ishikawa and HEC-1B endometrial cancer cell lines in a dosedependent manner. Obviously, compound 968 increased the sensitivity of the endometrial cancer cells to paclitaxel. Increased ROS production was also observed with increasing doses of compound 968. Moreover, compound 968 inhibited cell invasion in a dose-dependent manner. In vivo, compound 968 significantly reduced tumor growth in Ishikawa xenografts. These results support that compound 968 is a promising anti-tumorigenic agent and that combination with paclitaxel may be a valuable strategy for the treatment of endometrial cancer.

In acute myeloid leukemia, it has been demonstrated that GLS1 inhibition can induce mitochondrial apoptosis, suggesting that the pro-apoptotic pathway is downstream of GLS1 disruption[21]. Our results showed that compound 968 inhibited cell growth by promoting G1 phase cell cycle arrest, augmented Annexin-V expression, decreased the expression of cyclin D1 and MCL-1, and increased p21 and p27 expression in the two cell lines tested. These results are consistent with experiments showing that deprivation of glutamine induces apoptosis and cell cycle arrest in certain cancer types[22].

The effects of ROS imbalance on tumor growth are complex. Elevated levels of ROS have been linked to tumor progression, while the increased activity of antioxidant enzymes can enable cancer cells to tolerate higher levels of ROS[23]. Increased ROS levels affect several aspects of tumor biology. Importantly, they induce DNA damage and genomic instability[24]. Glutaminase has been directly linked to the redox balance in cancer cells $[25,26]$. The deprivation of glutamine in cell culture can increase ROS production and induce endoplasmic reticulum stress[6]. One of the major intracellular antioxidants is glutathione (GSH), a tripeptide of glutamate, cysteine, and glycine that is influenced by the abundance of glutamine and the activity of glutaminase. It has been shown that the GSH level is decreased in GLS-silenced glioma cells[27]. Weiss and his group showed that glutaminase inhibition in renal cancer cells exhibited apoptosis associated with a decreased GSH/GSSG ratio[28]. Treatment of lung cancer cells with BPTES, an allosteric inhibitor of glutaminase, resulted in increased ROS levels through the inhibition of glucose flow into the Krebs cycle and reduced oxidative phosphorylation in the mitochondria[29]. In agreement with previous studies of glutamine metabolism in other cancers, we found that compound 968 treatment led to decreased GLS1 expression and increased ROS production in a dose-dependent manner, 
accompanied by increased expression of ER stress markers, including Calnexin, BIP, and PERK. The complexity of cellular oxidative stress and endoplasmic reticulum stress and the exact mechanisms by which compound 968 contributes to both types of stress provide opportunities for further investigation.

Epithelial-to-mesenchymal transition (EMT) plays a critical role in tumor metastasis, which is the most common cause of cancer-related death. EMT is involved in critical phenotypic changes that improve mesenchymal migration and invasion, including the loss of E-cadherin expression in epithelial tissue and the increased expression of vimentin in mesenchymal tissues[30]. The reduction of E-cadherin expression is considered an essential event during EMT. It has been shown that the treatment of breast cancer cells with compound 968 resulted in reduced expression of genes involved in cell migration, invasion, and metastasis[22]. One study revealed that inhibition of glutamine metabolism by GLS1-targeting shRNA or GLS inhibitors (compound 968 or BPTES) diminished TGF- $\beta$-induced EMT and E-cadherin downregulation[31]. Recently, glutamine has been shown to promote the invasion of ovarian cancer cells. GLS1-targeted siRNA significantly decreased cancer growth and invasion in a mouse model of ovarian cancer[25]. In our study, the treatment of endometrial cancer cells with compound 968 for $24 \mathrm{~h}$ impaired cell invasion, which was accompanied by the increased expression of E-cadherin and the decreased expression of $\beta$-catenin and vimentin.

mTOR is a master regulator of cancer cell metabolism, controlling the synthesis of protein, lipids, and nucleotides, which can be activated by glutaminolysis[20]. In our previous study, glutamine was shown to promote ovarian cancer cell proliferation through the mTOR/S6 pathway[6]. In the present study, GLS1 inhibition in Ishikawa and HEC-1B cells using compound 968 exhibited obvious inhibition of P-S6K1, which is a marker of mTORC1 activity; this finding is consistent with our previous experimental results. In breast cancer cell lines, the GLS1 inhibitor CB-839 has also been demonstrated to reduce the levels of S6K1 and its downstream P-S6[32]. Furthermore, after cells were treated with compound 968, the phosphorylation of AKT at Ser473, which is another indicator of mTORC2 activity, was also decreased. Thus, the anti-tumorigenic effects of compound 968 are dependent on the mTOR signaling pathway. Previous studies indicated mTOR as a major inhibitor of autophagy induction[18]. Our data showed that mTOR inhibition by compound 968 induced autophagy, resulting in an increase in LC3 and Beclin-1 in endometrial cancer cells. Several studies showed that the induction of autophagy correlates with the development of endometrial cancer under hypoxia or nutrient deprivation conditions[19]. Hence, we hypothesize that compound 968 may also be able to potentiate its anti-proliferative effect by inducing autophagy in endometrial cancer.

Early strategies for targeting glutamine metabolism in cancer cells involving glutamine mimetic antimetabolites, such as azaserine and 6-diazo-5-oxo-L-norleucine (DON), had yielded promising preclinical data. However, these molecules failed to exhibit sufficient therapeutic effects in clinical trials due to excessive gastrointestinal toxicity and neurotoxicity[33]. Currently, several ongoing phase-I clinical trials are evaluating the safety, pharmacokinetics, and pharmacodynamics of GLS1 inhibitors, including CB-839, in patients with hematological malignancies and solid tumors, such as breast cancer, lung cancer and renal cancer (www.clinicaltrials.gov). Although compound 968 has been demonstrated to have little 
to no effect on normal cells in vivo, its safety and clinical efficacy in human cancers need to be evaluated in future clinical trials[4]. High expression of GLS1 has been associated with paclitaxel resistance in breast cancer cells, and GLS1 silencing rescued the sensitivity of paclitaxel-resistant breast cancer cells to paclitaxel[34]. Our studies found that the combination of compound 968 and paclitaxel synergistically increased the sensitivity of endometrial cancer cell lines to paclitaxel. Thus, the combination of GLS1 inhibitors with conventional chemotherapy may be more effective in cancer treatment.

\section{Conclusion}

Taken together, the elevated expression of GLS1 in endometrial cancer tissues provides a strong rationale for GLS1 inhibition in this tumor entity. Targeting GLS1 via compound 968 has promising antitumorigenic effects in endometrial cancer cell lines and xenografts. Therefore, we propose that targeting glutamine metabolism by GLS1 inhibition may act as a novel approach for the management of human endometrial cancer.

\section{Abbreviations}

CCLE: Cancer Cell Line Encyclopedia; TCGA: The Cancer Genome Atlas; GEPIA: Gene Expression Profiling Interactive Analysis; OS: Overall survival; PFS: progression-free survival; P-AKT473: phosphoAKT(Ser473); P-S6: phospho-S6 ribosomal; TCA: tricarboxylic acid; UCEC: uterine corpus endometrial carcinoma; GLS1: glutaminase-1 enzyme; a-KG: a-ketoglutarate; MTT: 3-(4,5-dimethyl-2-thiazolyl)-2,5diphenyl-2H-tetrazolium bromide; PBS: phosphate - buffered saline; PI: propidium iodide; ROS: reaction oxygen species; TNBC: triple-negative breast cancer; PERK: protein kinase-like endoplasmic reticulum kinase; BIP: binding immunoglobulin protein; LC3: light chain 3; EMT: epithelial-mesenchymal transition; DON: 6-diazo-5-oxo-L-norleucine;

\section{Declarations}

\section{Acknowledgements}

Not applicable.

\section{Author contributions}

GH and YLQ performed the experiments and wrote the paper. PGY, WC, LDP and LNF analyzed the data. WL and LPP performed experiments. SXG and YLQ conceived the study, designed the experiments and revised the manuscript. All authors read and approved the final manuscript.

\section{Funding}

This work was supported by the Shandong Academy of Medical Science Found (2016-22, Yuan L). This work was also supported by National Natural Science Foundation of China (81672591, Sheng X), 
Shandong Provincial Key Research and Development Program Fund (2016GSF201211, Wei L) and Shandong Health Department Fund (2015WS0155 and 2016WS0554, Wei L).

\section{Availability of data and materials}

The data supporting the conclusions of this paper are included within the manuscript.

\section{Ethics approval and consent to participate}

All animal procedures were performed under the guidelines of the institutional review board and the ethics committee of Shandong Cancer Hospital and Institute.

\section{Consent for publication}

All the authors agree to the publication clause.

\section{Competing interests}

The authors declare no competing interests.

\section{References}

1. Lortet-Tieulent JFJ, Bray F, Jemal A. International Patterns and Trends in Endometrial Cancer Incidence, 1978-2013. J Natl Cancer Inst. 2018;110:354-61.

2. Mates JM, Di Paola FJ, Campos-Sandoval JA, Mazurek S, Marquez J. Therapeutic targeting of glutaminolysis as an essential strategy to combat cancer. Semin Cell Dev Biol. 2019.

3. Yang L, Venneti S, Nagrath D. Glutaminolysis. A Hallmark of Cancer Metabolism. Annu Rev Biomed Eng. 2017;19(1):163-94.

4. Wang JBEJ, Fuji R, Ramachandran S, Gao P, Dinavahi R, et al. Targeting mitochondrial glutaminase activity inhibits oncogenic transformation. Cancer Cell. 2010;18:207-19.

5. Duran RV, Oppliger W, Robitaille AM, Heiserich L, Skendaj R, Gottlieb E, Hall MN. Glutaminolysis activates Rag-mTORC1 signaling. Mol Cell. 2012;47(3):349-58.

6. Yuan LQSX, Willson AK, Roque DR, Stine JE, Guo H, et al. Glutamine promotes ovarian cancer cell proliferation through the mTOR/S6 pathway. Endocr Relat Cancer. 2015;22:577-91.

7. Thangavelu K, Pan CQ, Karlberg T, Balaji G, Uttamchandani M, Suresh V, Schuler H, Low BC, Sivaraman J. Structural basis for the allosteric inhibitory mechanism of human kidney-type glutaminase (KGA) and its regulation by Raf-Mek-Erk signaling in cancer cell metabolism.

Proceedings of the National Academy of Sciences. 2012;109(20):7705-7710.

8. Altman BJ, Stine ZE, Dang CV. From Krebs to clinic: glutamine metabolism to cancer therapy. Nat Rev Cancer. 2016;16:619-34.

9. Dang C. MYC. microRNAs and glutamine addiction in cancers. Cell Cycle. 2014;8:3243-5. 
10. Speck RFZL, Huang Y, Tian CH, Taylor L, Curthoys N, et al. Interferon-a Regulates Glutaminase 1 Promoter through STAT1 Phosphorylation: Relevance to HIV-1 Associated Neurocognitive Disorders. PLoS ONE. 2012;7:e32995.

11. Akins NSNT, Le HV. Inhibition of Glycolysis and Glutaminolysis: An Emerging Drug Discovery Approach to Combat Cancer. Curr Top Med Chem. 2018;18:1-11.

12. Szeliga MB-KM, Rozycka A, Hilgier W, Marquez J, Albrecht J. Silencing of GLS and overexpression of GLS2 genes cooperate in decreasing the proliferation and viability of glioblastoma cells. Tumour Biol. 2014;35:1855-62.

13. Yu DSX, Meng G, Chen J, Yan C, Jiang Y, et al. Kidney-type glutaminase (GLS1) is a biomarker for pathologic diagnosis and prognosis of hepatocellular carcinoma. Oncotarget. 2015;6:7619-31.

14. Yuan LQSX, Clark LH, Zhang L, Guo H, Jones HM, et al. Glutaminase inhibitor compound 968 inhibits cell proliferation and sensitizes paclitaxel in ovarian cancer. Am J Transl Res. 2016;8:4265-77.

15. Katt WPAM, Cerione RA. Simultaneously targeting tissue transglutaminase and kidney type glutaminase sensitizes cancer cells to acid toxicity and offers new opportunities for therapeutic intervention. Mol Pharm. 2015;12:46-55.

16. Hui Guo YZ, Amanda L, Jackson, Leslie H, Clark J, Kilgore Lu, Zhang J, Han X, Sheng TP, Gilliam PA, Gehrig. Chunxiao Zhou, Victoria L. Bae-Jump Everolimus exhibits anti-tumorigenic activity in obesityinduced ovarian cancer Oncotarget. 2016;7(15):20338-20356.

17. Halama AKM, Dib SS, Zaghlool SB, Siveen KS, Iskandarani A, et al. Accelerated lipid catabolism and autophagy are cancer survival mechanisms under inhibited glutaminolysis. Cancer Lett. 2018;430:133-47.

18. Moncada SHE, Colombo SL. Fulfilling the metabolic requirements for cell proliferation. Biochem J. 2012;446:1-7.

19. Doria AGM, Punzi L. Autophagy in human health and disease. N Engl J Med. 2013;368:1845.

20. Lheureux SOA. Endometrial cancer-targeted therapies myth or reality? Review of current targeted treatments. European Journal of Cancer. 2016;59:99-108.

21. Jacque NRA, Larrue C, Meunier G, Birsen R, Willems $L$, et al. Targeting glutaminolysis has antileukemic activity in acute myeloid leukemia and synergizes with BCL-2 inhibition. Blood. 2015;126:1346-56.

22. Simpson NETV, Pogribna M, Beland FA, Pogribny IP. Modifying metabolically sensitive histone marks by inhibiting glutamine metabolism affects gene expression and alters cancer cell phenotype. Epigenetics. 2014;7:1413-20.

23. Gius DSD. Redox signaling in cancer biology. Antioxid Redox Signal. 2006;8:1249-52.

24. Turgeon MOPNS, Poulogiannis G. DNA Damage, Repair, and Cancer Metabolism. Frontiers in Oncology. 2018;8.

25. Yang LMT, Mangala LS, Marini J, Zhao H, Wahlig S, et al. Metabolic shifts toward glutamine regulate tumor growth, invasion and bioenergetics in ovarian cancer. Molecular Systems Biology. 
2014;10:728.

26. Matés JMSJ, Martín-Rufián M, Campos-Sandoval JA, Alonso FJ, Márquez J. Glutaminase Isoenzymes as Key Regulators in Metabolic and Oxidative Stress Against Cancer. Curr Mol Med. 2013;13:514-34.

27. Martin-Rufian MN-GR, Higuero A, Crisma AR, Campos-Sandoval JA, Gomez-Garcia MC, et al. Both GLS silencing and GLS2 overexpression synergize with oxidative stress against proliferation of glioma cells. J Mol Med (Berl). 2014;92:277-90.

28. Aboud OAHS, Trott J, Stewart B, Liang S, Chaudhari AJ, et al. Glutamine Addiction in Kidney Cancer Suppresses Oxidative Stress and Can Be Exploited for Real-Time Imaging. Can Res. 2017;77:674658.

29. Ulanet DBCK, Jha A, Choe S, Wang A, Woo H, et al. Mesenchymal Phenotype Predisposes Lung Cancer Cells to Impaired Proliferation and Redox Stress in Response to Glutaminase Inhibition. PLoS ONE. 2014;9:e115144.

30. Puisieux ABT, Caramel J. Oncogenic roles of EMT-inducing transcription factors. Nat Cell Biol. 2014;16:488-94.

31. Lee SYJH, Ju MK, Jeong EK, Kim CH, Park HG, et al. Dlx-2 and glutaminase upregulate epithelialmesenchymal transition and glycolytic switch. Oncotarget. 2016;7:7925-39.

32. Lampa MAH, He T, Ospina B, Reeves J, Zhang B, et al. Glutaminase is essential for the growth of triple-negative breast cancer cells with a deregulated glutamine metabolism pathway and its suppression synergizes with mTOR inhibition. PLoS One. 2017;12:e0185092.

33. Ahluwalia GSGJ, Hao Z, Cooney DA. Metabolism and action of amino acid analog anti-cancer agents. Pharmacol Ther. 1990;46:243-71.

34. Fu AYZ, Song Y, Zhang E. Silencing of glutaminase 1 resensitizes Taxol-resistant breast cancer cells to Taxol. Mol Med Rep. 2015;11:4727-33.

\section{Figures}


a

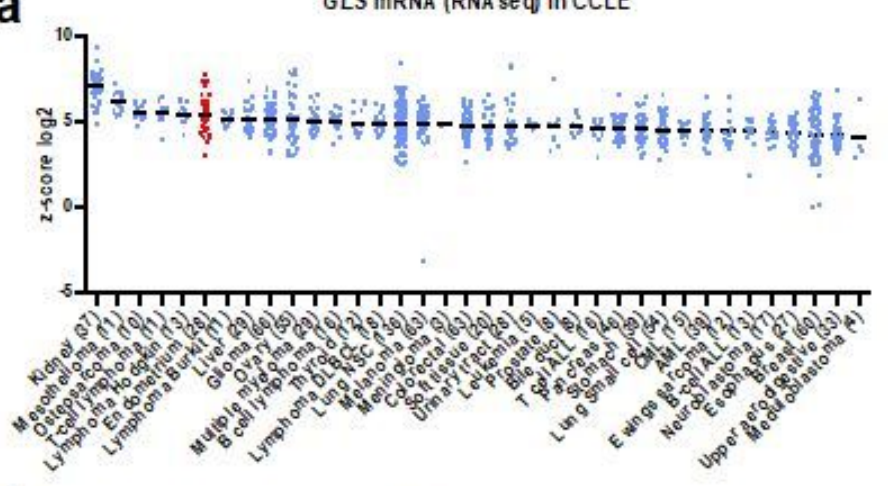

b

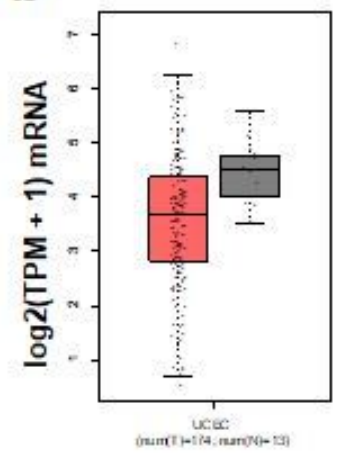

C

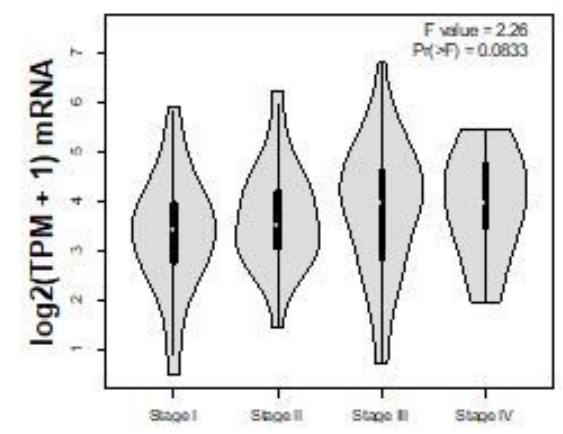

d

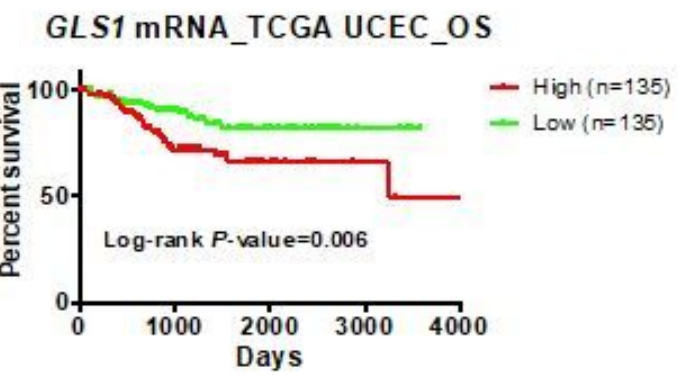

e

GLS1 MRNA_TCGA UCEC_PFS

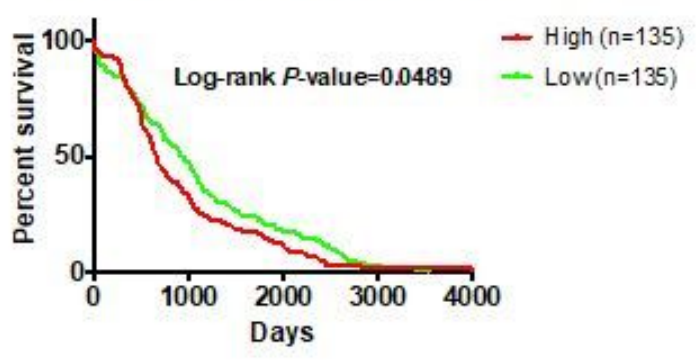

\section{Figure 1}

GLS1 expression is elevated during endometrial cancer progression and associates with poor prognosis. a GLS1 mRNA expression in 877 cancer cell lines (data extracted from microarray data of CCLE). GLS1 expression is elevated in endometrial cell lines. b GLS1 expression in tumor (red) and related normal tissue (grey) in uterine corpus endometrial cancer (UCEC; TCGA) RNA sequencing data; data accessed through the gene expression viewer of Firebrowse, and are represented as log2(TPM + 1) mRNA levels. $c$ Pathological Stage Plot for GLS1 expression in uterine corpus endometrial cancer (UCEC; TCGA). GLS1 expression is elevated during endometrial cancer progression. $d$, e Kaplan-Meier survival plot showing that high levels of GLS1 mRNA are significantly associated with reduced patient overall survival and progression-free survival in uterine corpus endometrial carcinoma patients $(n=527$; data retrieved from UCEC TCGA, PanCancer Atlas RNA sequencing data using Broad GDAC Firehose). Red = High expression, green = low expression; divided at median. 
a

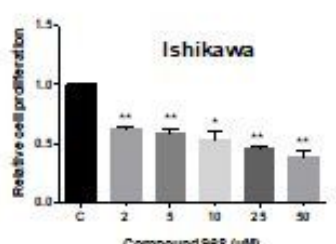

b

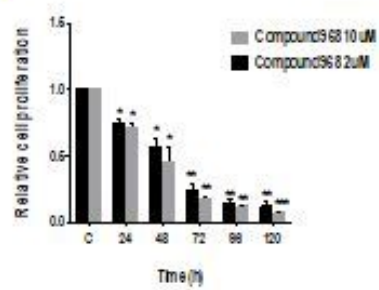

c

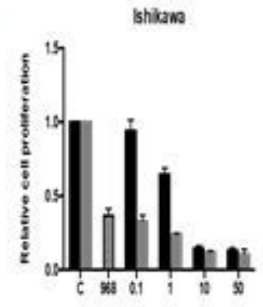

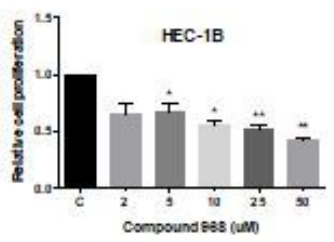

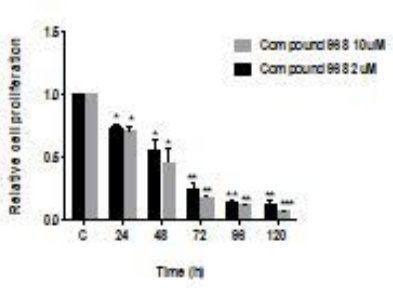

Hec-1B
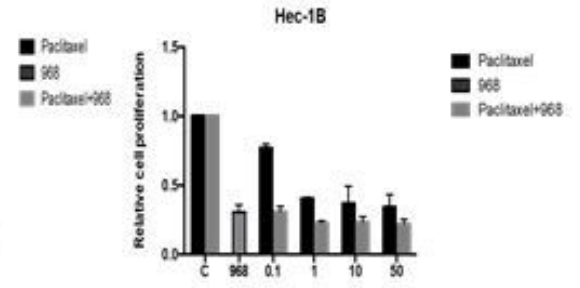

d

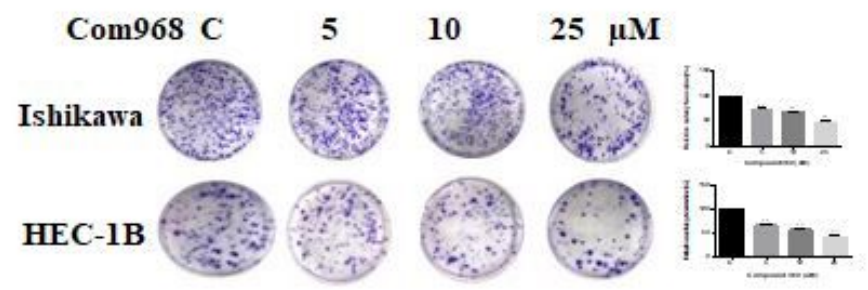

e

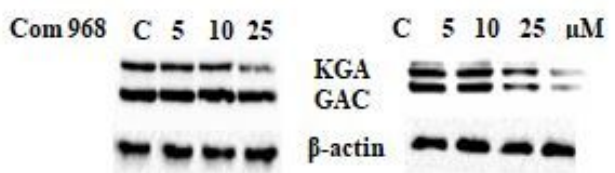

HEC-1B

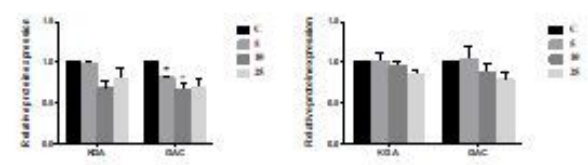

Figure 2

Compound 968 inhibits the proliferation of endometrial cancer cells. a, b Ishikawa and HEC-1B cells were cultured for $5 \mathrm{~h}$ and then treated with varying concentrations of compound 968 for $72 \mathrm{~h}$. Alternatively, cells were treated with $2 \mu \mathrm{M}$ or $10 \mu \mathrm{M}$ for different times ( $24 \mathrm{~h}, 48 \mathrm{~h}, 72 \mathrm{~h}, 96 \mathrm{~h}$, and $120 \mathrm{~h}$ ). c The cells were treated with $10 \mathrm{~mm}$ compound 968 in combination with different concentrations of paclitaxel for 72h. $\mathrm{d}$ The effects of compound 968 on the long-term growth of Ishikawa and HEC-1B were assessed through a colony-forming assay. e Compound 968 reduced GLS1 (KGA and GAC) expression after $24 \mathrm{~h}$ treatment. Data were shown as the mean \pm SEM of two experiments; ${ }^{*} \mathrm{P}<0.05,{ }^{*} \mathrm{P}<0.01$. 
a

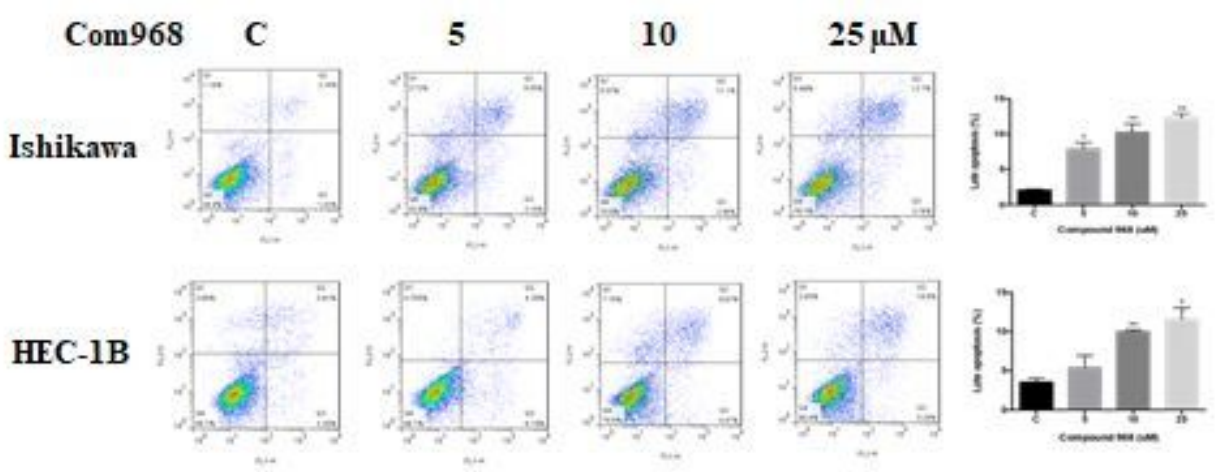

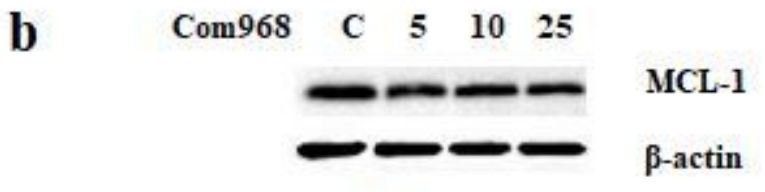

Ishikawa

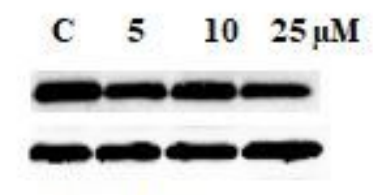

HEC-1B

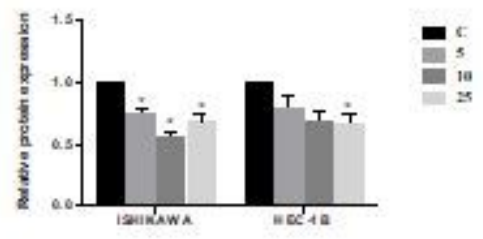

Figure 3

Compound 968 induces cell apoptosis in endometrial cancer cells. a Apoptosis was detected using the Annexin-V FITC assay. Compound 968 increased Annexin V levels in these two cell lines. b Western blotting indicated that treatment with compound 968 for $24 \mathrm{~h}$ decreased the expression of the antiapoptotic protein MCL-1. *P $<0.05$. 
a
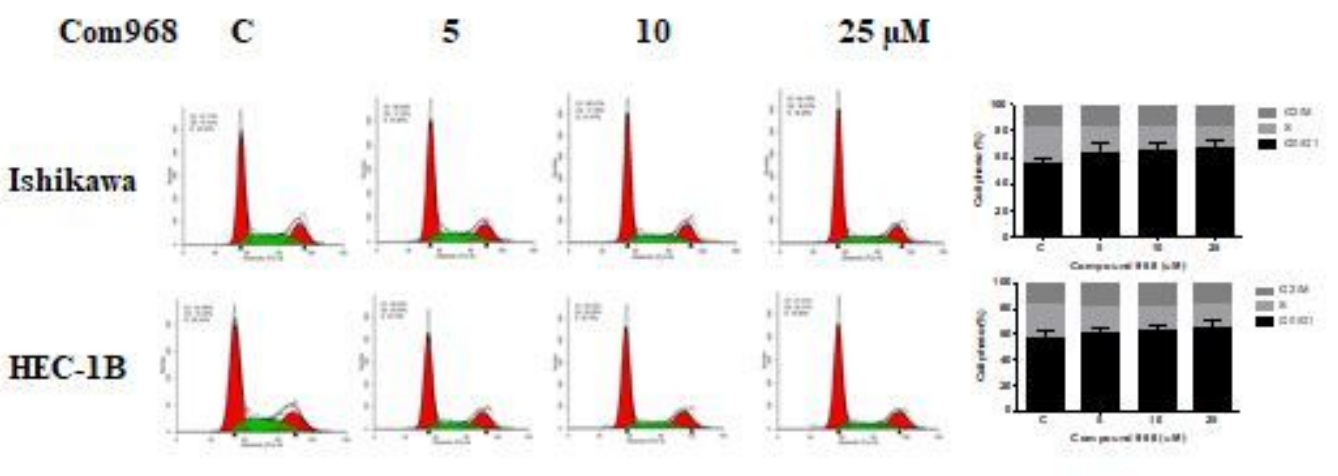

b
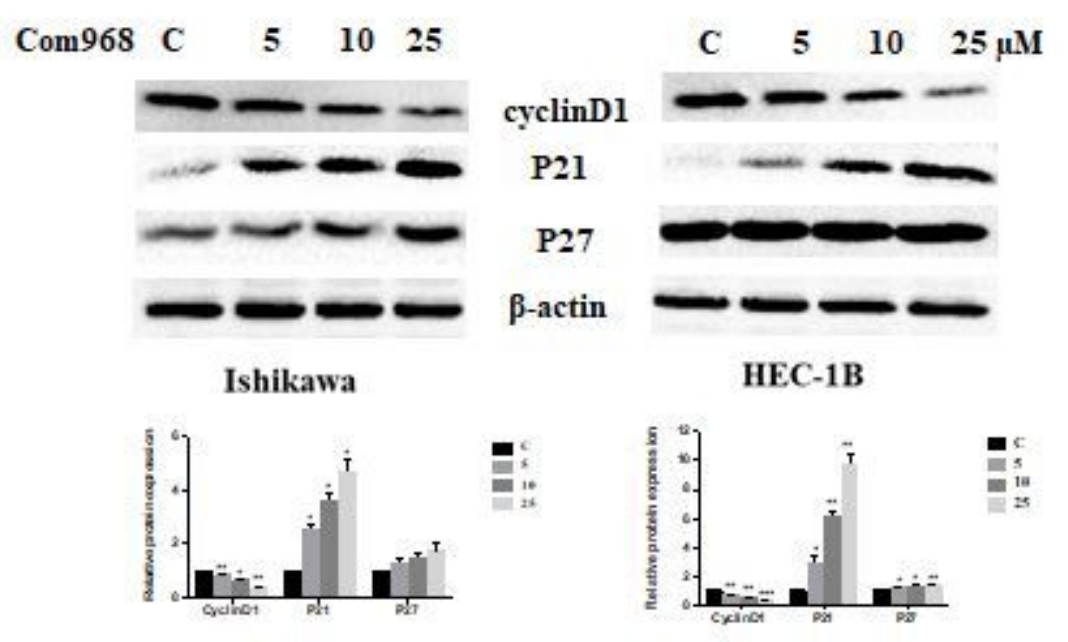

\section{Figure 4}

Compound 968 induces cell cycle G1 phase arrest. a Compound 968 markedly induced cell cycle G1 arrest in both cell lines in a dose-dependent manner, and this effect was accompanied by a decrease in the percentage of cells in S-phase cells. b Western blotting indicated that compound 968 decreased the expression of cyclin D1 and increased the expression of p21 and p27 in the Ishikawa and HEC-1B cells; $\star P<0.05, * * P<0.01$. 
a

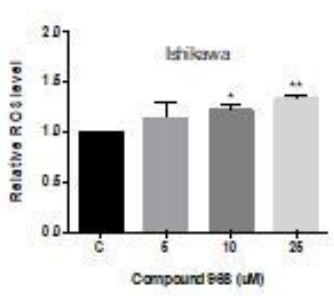

b

$\begin{array}{llllll}0 & 4 & 8 & 12 & 16 & 24 \mathrm{H}\end{array}$

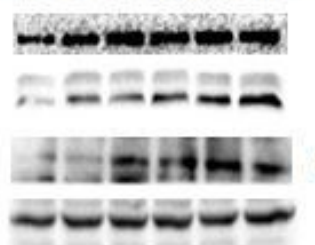

Ishikawa

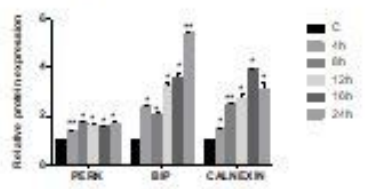

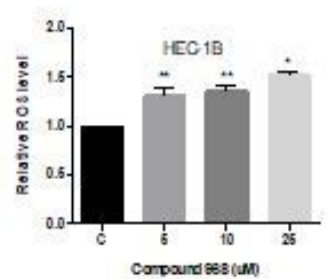

$\begin{array}{lllllll}0 & 4 & 8 & 12 & 16 & 24 \mathrm{H}\end{array}$

PERK

BIP

Calnexin

$\beta$-actin

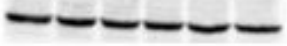

HEC-1B

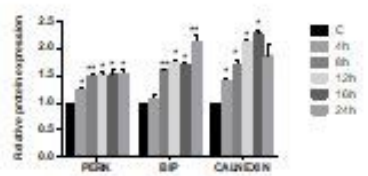

C

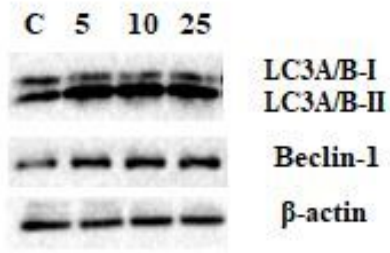

Ishikawa
$\begin{array}{llll}C & 5 & 10 & 25 \mu \mathrm{M}\end{array}$

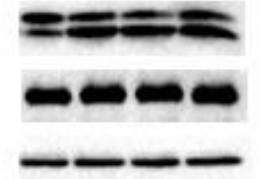

HEC-1B
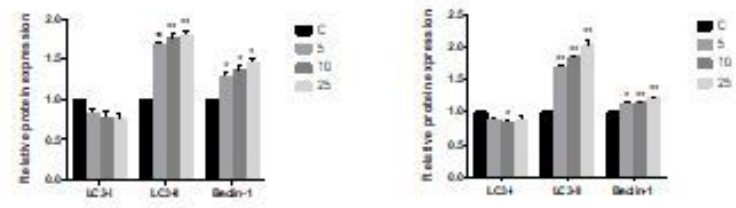

\section{Figure 5}

Compound 968 induces oxidative stress and autophagy in endometrial cancer cells. a Intracellular ROS production was tested using the DCFH-DA assay. Compound 968 increased the ROS levels in a dosedependent manner. $b$ The cells were treated with compound $968(10 \mu \mathrm{M})$ for different time points, and western blotting showed that compound 968 increased the protein levels of stress proteins (PERK, BIP, and Calnexin) in a time-dependent manner in Ishikawa and HEC-1B cells. c Autophagy in the Ishikawa and HEC-1B cell lines was analyzed by immunoblotting after treatment with compound 968 for $24 \mathrm{~h}$. Compound 968 increased the protein levels of LC3 and Beclin- $1 ; * P<0.05, * * P<0.01$. 
a

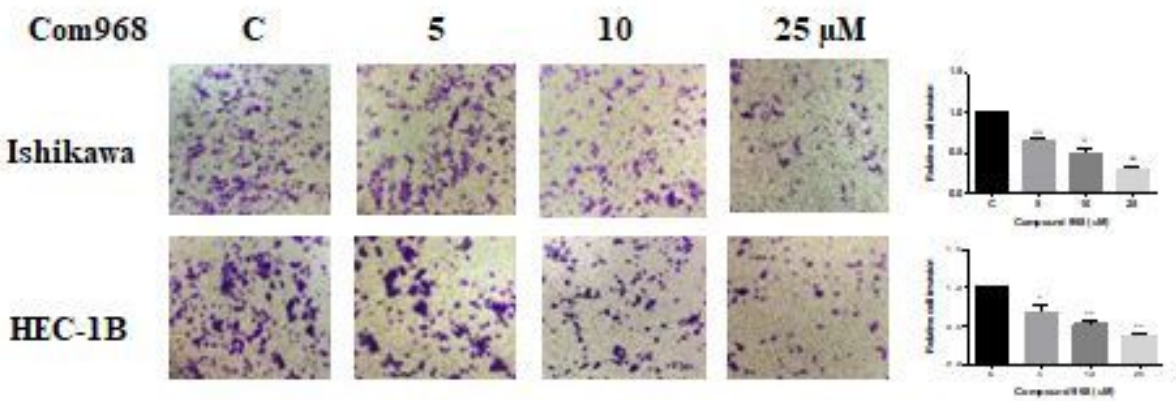

b

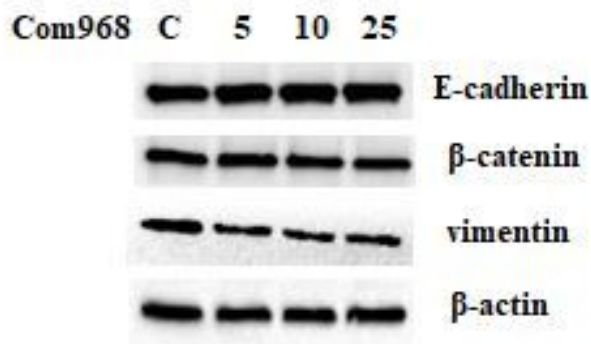

Ishikawa

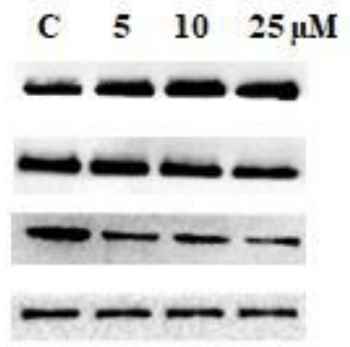

HEC-1B
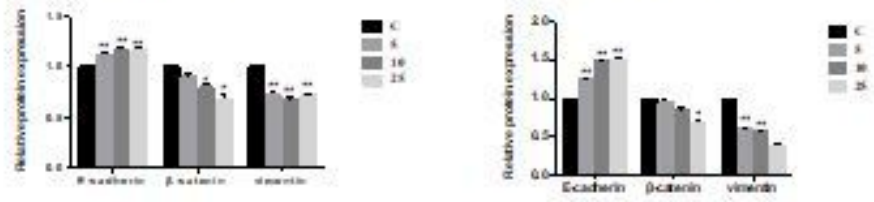

Figure 6

Compound 968 inhibits cell invasion in endometrial cancer cells. a Compound 968 reduced cell invasion in both cell lines. b Western blotting showed that compound 968 treatment resulted in increased Ecadherin protein levels, while $\beta$-catenin and vimentin protein levels were decreased; ${ }^{*} P<0.05, * \star P<0.01$.

a

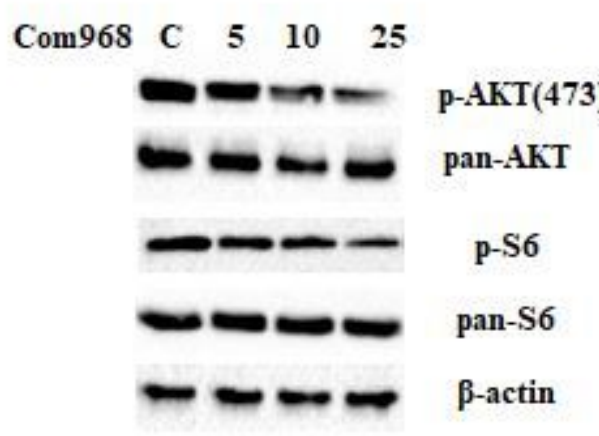

C $\quad 5 \quad 10 \quad 25 \mu M$

Ishikawa

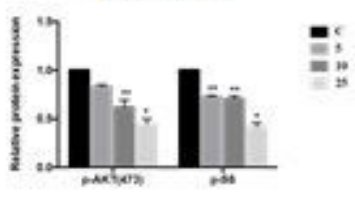

HEC-1B

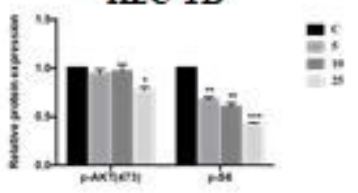


Figure 7

Effects of compound 968 on AKT/mTOR/S6 pathway in endometrial cancer cells. a p-AKT473 and p-S6 were assessed by western blotting. Compound 968 inhibited the activity of the AKT/mTOR /S6 pathway in both cell lines; ${ }^{*} \mathrm{P}<0.05,{ }^{* *} \mathrm{P}<0.01$.

a
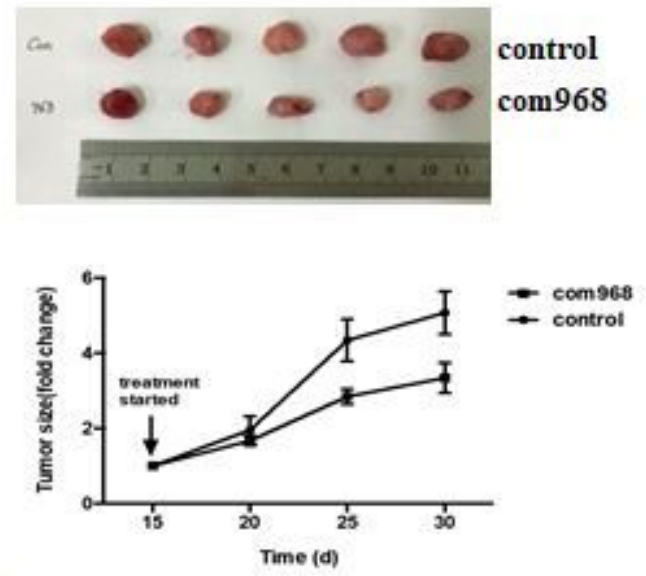

b

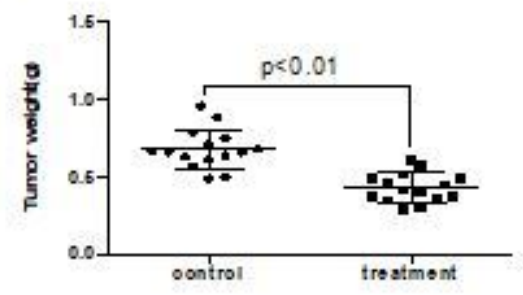

c

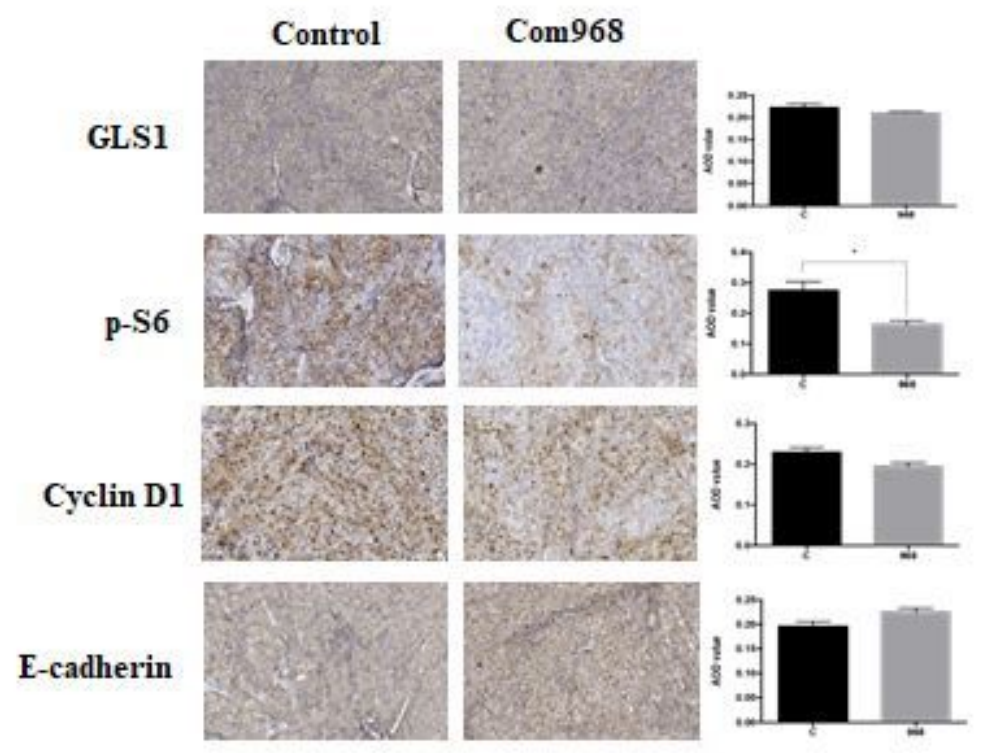

\section{Figure 8}

Compound 968 inhibits tumor growth in vivo. a, b Compound 968 significantly decreased tumor weight and volume. c IHC showed that GLS1, cyclinD1 and p-S6 expression was decreased by compound 968 treatment. E-cadherin expression was enhanced after compound 968 treatment. ${ }^{*} P<0.05$. 